\title{
Cryo-STEM EELS of Nafion Saturated with an Organic Solvent
}

Sergey Yakovlev and Matthew Libera

Department of Chemical Biomedical Materials Engineering, Stevens Institute of Technology, Hoboken, NJ, USA, 07002

Nafion is a perfluorinated ionomer that is often used as a membrane material in fuel-cell applications and in perm-selective clothing applications where moisture and air must be allowed to pass while toxic substances must be blocked. At the molecular level, Nafion consists of a perfluorinated carbon main chain with perfluoroether side chains terminated by sulfonate groups. This molecular architecture is believed to give rise to a nanophase-separated (5-20 nm length scales) morphology consisting of interpenetrated hydrophobic and hydrophilic channels through which water and organic solvents, respectively, can be transported. Continued optimization of differential transport properties through Nafion requires a more precise determination of its nanoscale morphology, and, while there are many techniques to determine the total amount of solvent dissolved in such polymers, spatially resolved electron energy-loss spectroscopy (EELS) in the STEM brings an unchallenged combination of spectral sensitivity and spatial resolution to determining polymer morphology at nano and micro length scales [1]. Techniques to map water in frozen-hydrated tissue [2] and polymers [3, 4] have already been developed, and colloidal specimens preserved in frozen organic solvents have been imaged using bright-field cryo TEM techniques [5]. By combining these approaches, we are working to develop methods to spectroscopically image solvated polymers where the spatial distribution of solvent is controlled by the underlying nanoscale polymer morphology.

Because the ultimate spatial resolution of spectroscopic imaging of radiation-sensitive materials is ultimately controlled by the incident electron dose [3], we work with low-loss spectra where the scattering cross sections are substantially higher than in the core loss region. Figure 1 shows lowloss EELS spectra characteristic of Nafion, amorphous ice, and frozen dimethyl methylphosphonate (DMMP), a moderately polar organic solvent which is used to simulate sarin nerve agent in permselective clothing applications. Importantly we find that liquid DMMP can be quenched using traditional cryo techniques to form electron-transparent solid films appropriate for TEM [6]. We see from figure 1 that the low-loss spectra characteristic of Nafion, water, and DMMP are distinguishable from each other, both in the region of the plasmon peaks $(19-23 \mathrm{eV})$ and in the fine structure on the rising edge of the plasmon peaks. As a first test of our ability to quantify solvated composition using EELS, we used collected a spectrum from a thin section cryo-microtomed from a thick film of Nafion fully saturated with DMMP, and we used Multiple Least Squares fitting [7] to fit this spectrum with a linear combination of reference spectra taken from pure components. MLS fitting indicates that this specimen contains $53 \%$ Nafion in very good agreement with the value of $55 \%$ determined by a weight-gain measurement.

Fluorinated polymers are particularly sensitive to electron-beam damage (8), and we observe that dry Nafion rapidly loses mass under $200 \mathrm{keV}$ electron irradiation. Figure 3 shows the results of a $\tau / \lambda$ analysis of spectra collected at $-165^{\circ} \mathrm{C}$ from dry Nafion. While we observe some modifications to the low-loss spectra, the decrease in specimen thickness is profound, and we attribute this to chain scission events followed by sublimation of volatile polymer fragments. Significantly, the dose- 
dependent decrease in specimen thickness associated with the study of a frozen DMMP - saturated Nafion specimen (figure 3) is orders of magnitude less than that of dry Nafion, and we speculate that the apparent protection is due to the fact that sublimation of chain scission products is limited by the requirement to simultaneously sublimate the frozen solvent. We observe a similar, though somewhat less pronounced, solvo-protection effect when studying hydrated Nafion.

\section{References}

1. M. Libera and M. Disko, Electron Energy Loss Spectroscopy of Polymer, in Transmission EELS in Material Science and EELS Atlas, 2th ed., WILEY_VCH Verlag GmbH \& Co KGaA Weinheim, 2004.

2. R. D. Leapman and S. Sun, Ultramicroscopy, Volume 59, Issues 1-4, July 1995, Pages 71-79.

3. A. Sousa, A. Aitouchen and M. Libera, Ultramicroscopy, Volume 106, Issue 2, January 2006, Pages 130-145.

4. G. Kim, A. Sousa, et al. submitted to JACS (2006).

5. D. Danino, R. Gupta, J. Satyavolu and Y. Talmon, Journal of Colloid and Interface Science 249 (1), pp. $180-186$.

6. J. Dubochet, M. Adrian, J. Lepault and A. W. McDowall Dubochet, Trends in Biochemical Sciences, Volume 10, Issue 4, April 1985, Pages 143-146.

7. R. D. Leapman R. L. Ornberg Ultramicroscopy, Volume 24, Issues 2-3, 1988, Pages 251-268.

8. B. J. Ciliax, K. L. Kirk and R. D. Leapman, Ultramicroscopy, Volume 48, Issues 1-2, January 1993, Pages 13-25.

9. Research support provided by the Army Research Office (Grant \#DAAD19-03-1-0271).

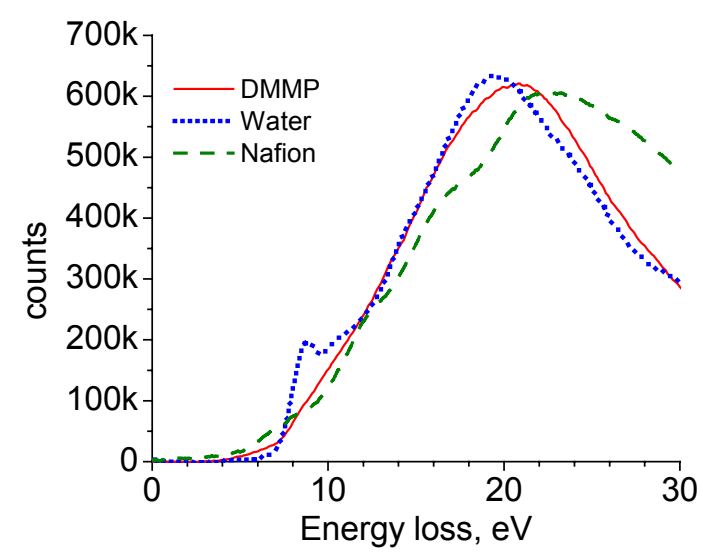

Figure 1. Energy loss spectra of DMMP, water, and Nafion

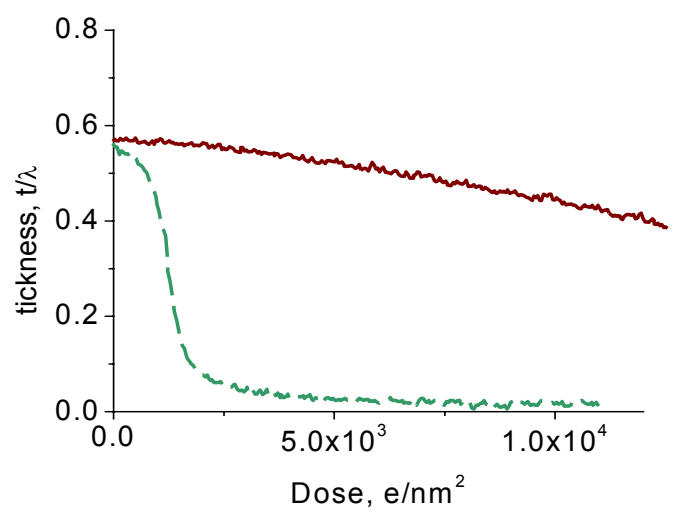

Figure 3. Thickness change under electron beam irradiation (dashed line - dry Nafion, solid line - saturated with $55 \%$ of DMMP). Beam size $200 \mathrm{~nm}$.

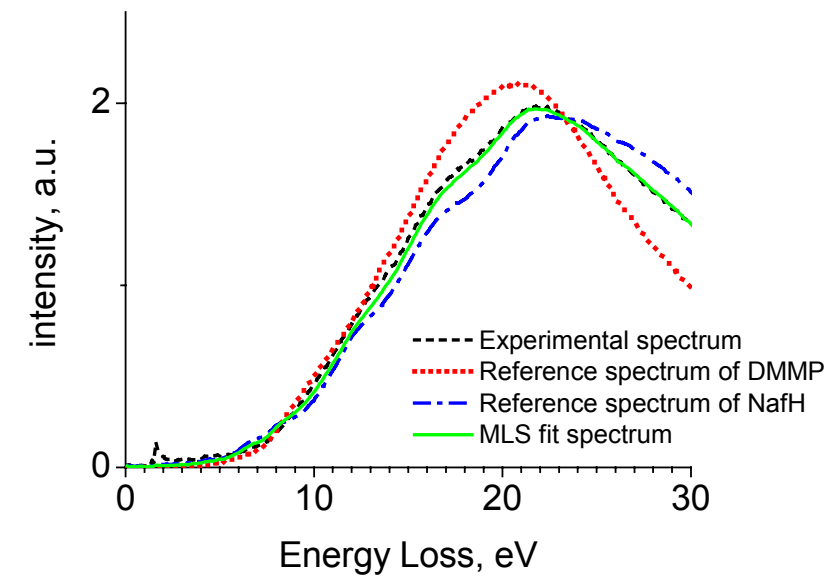

Figure 2. MLS fitting for Nafion saturated with $55 \%$ of DMMP. Beam size $200 \mathrm{~nm}$. 